\title{
Nonperturbative transverse-momentum-dependent effects in dihadron and direct photon-hadron angular correlations in $p+p$ collisions at $\sqrt{s}=200 \mathrm{GeV}$
}

C. Aidala,${ }^{39}$ Y. Akiba,${ }^{50,51, \dagger}$ M. Alfred,${ }^{22}$ V. Andrieux,${ }^{39}$ N. Apadula,${ }^{27}$ H. Asano, ${ }^{32,50}$ B. Azmoun, ${ }^{7}$ V. Babintsev,,${ }^{23}$ A. Bagoly, ${ }^{16}$ N. S. Bandara ${ }^{38}$ K. N. Barish, ${ }^{8}$ S. Bathe,${ }^{5,51}$ A. Bazilevsky, ${ }^{7}$ M. Beaumier, ${ }^{8}$ R. Belmont, ${ }^{12}$ A. Berdnikov, ${ }^{53}$ Y. Berdnikov, ${ }^{53}$ D. S. Blau, ${ }^{31,42}$ M. Boer, ${ }^{34}$ J. S. Bok ${ }^{44}$ M. L. Brooks, ${ }^{34}$ J. Bryslawskyj, ${ }^{5,8}$ V. Bumazhnov, ${ }^{23}$ S. Campbell, ${ }^{13}$ V. Canoa Roman, ${ }^{56}$ R. Cervantes, ${ }^{56}$ C. Y. Chi, ${ }^{13}$ M. Chiu, ${ }^{7}$ I. J. Choi, ${ }^{24}$ J. B. Choi, ${ }^{10,}{ }^{*}$ Z. Citron, ${ }^{61}$ M. Connors, ${ }^{20,51}$ N. Cronin, ${ }^{56}$ M. Csanád, ${ }^{16}$ T. Csörgő, ${ }^{17,62}$ T. W. Danley, ${ }^{45}$ M. S. Daugherity, ${ }_{56}$ G. David, ${ }^{7,56}$ K. DeBlasio, ${ }^{43}$ K. Dehmelt ${ }^{56}$ A. Denisov, ${ }^{23}$ A. Deshpande, ${ }^{51,56}$ E. J. Desmond, ${ }^{7}$ A. Dion, ${ }^{56}$ D. Dixit, ${ }^{56}$ J. H. Do, ${ }^{63}$ A. Drees, ${ }^{56}$ K. A. Drees, ${ }^{6}$ J. M. Durham, ${ }^{34}$ A. Durum, ${ }^{23}$ A. Enokizono, ${ }^{50,52}$ H. En'yo, ${ }^{50}$ S. Esumi,${ }^{59}$ B. Fadem,${ }^{40}$ W. Fan, ${ }^{56}$ N. Feege,${ }^{56}$ D. E. Fields, ${ }^{43}$ M. Finger, ${ }^{9}$ M. Finger, Jr., ${ }^{9}$ S. L. Fokin, ${ }^{31}$ J. E. Frantz, ${ }^{45}$ A. Franz, ${ }^{7}$ A. D. Frawley, ${ }^{19}$ Y. Fukuda,${ }^{59}$ C. Gal,${ }^{56}$ P. Gallus, ${ }^{14}$ P. Garg,${ }^{3,56}$ H. Ge, ${ }^{56}$ F. Giordano, ${ }^{24}$ Y. Goto ${ }^{50,51}$ N. Grau, ${ }^{2}$ S. V. Greene, ${ }^{60}$ M. Grosse Perdekamp, ${ }^{24}$ T. Gunji, ${ }^{11}$

H. Guragain, ${ }^{20}$ T. Hachiya, ${ }^{41,50,51}$ J. S. Haggerty, ${ }^{7}$ K. I. Hahn, ${ }^{18}$ H. Hamagaki ${ }^{11}$ H. F. Hamilton, ${ }^{1}$ S. Y. Han, ${ }^{18}$ J. Hanks ${ }^{56}$ S. Hasegawa, ${ }^{28}$ T. O.S. Haseler, ${ }^{20}$ X. He ${ }^{20}$ T. K. Hemmick, ${ }^{56}$ J. C. Hill, ${ }^{27}$ K. Hill, ${ }^{12}$ A. Hodges, ${ }^{20}$ R. S. Hollis, ${ }^{8}$ K. Homma, ${ }^{21}$ B. Hong,${ }^{30}$ T. Hoshino, ${ }^{21}$ N. Hotvedt, ${ }^{27}$ J. Huang, ${ }^{7}$ S. Huang, ${ }^{60}$ K. Imai, ${ }^{28}$ M. Inaba ${ }^{59}$ A. Iordanova, ${ }^{8}$ D. Isenhower, ${ }^{1}$ D. Ivanishchev, ${ }^{49}$ B. V. Jacak, ${ }^{56}$ M. Jezghani, ${ }^{20}$ Z. Ji, ${ }^{56}$ X. Jiang, ${ }^{34}$ B. M. Johnson, ${ }^{7,20}$ D. Jouan, ${ }^{47}$

D. S. Jumper, ${ }^{24}$ J. H. Kang, ${ }^{63}$ D. Kapukchyan, ${ }^{8}$ S. Karthas, ${ }^{56}$ D. Kawall, ${ }^{38}$ A. V. Kazantsev, ${ }^{31}$ V. Khachatryan, ${ }^{56}$ A. Khanzadeev, ${ }^{49}$ C. Kim, ${ }^{8,30}$ E.-J. Kim, ${ }^{10}$ M. Kim ${ }_{54}^{5}$ D. Kincses, ${ }^{16}$ E. Kistenev, ${ }^{7}$ J. Klatsky, ${ }_{19}$ P. Kline ${ }^{56}$ T. Koblesky ${ }^{12}$ D. Kotov ${ }^{49,53}$ S. Kudo, ${ }^{59}$ B. Kurgyis, ${ }^{16}$ K. Kurita, ${ }^{52}$ Y. Kwon, ${ }^{63}$ J. G. Lajoie, ${ }^{27}$ A. Lebedev, ${ }^{27}$ S. Lee ${ }^{63}$ S. H. Lee, ${ }^{27,56}$ M. J. Leitch ${ }^{34}$ Y. H. Leung, ${ }^{56}$ N. A. Lewis, ${ }^{39}$ X. Li ${ }^{34}$ S. H. Lim, ${ }^{34,63}$ M. X. Liu, ${ }^{34}$ V.-R. Loggins, ${ }^{24}$ S. Lökös, ${ }^{16,17}$ K. Lovasz, ${ }^{15}$ D. Lynch, ${ }^{7}$ T. Majoros ${ }^{15}$ Y. I. Makdisi, ${ }^{6}$ M. Makek, ${ }^{64}$ V. I. Manko, ${ }^{31}$ E. Mannel, ${ }^{7}$ M. McCumber, ${ }^{34}$ P. L. McGaughey, ${ }^{34}$ D. McGlinchey, ${ }^{12,34}$ C. McKinney, ${ }^{24}$ M. Mendoza, ${ }^{8}$ A. C. Mignerey,${ }^{37}$ D. E. Mihalik, ${ }^{56}$ A. Milov, ${ }^{61}$ D. K. Mishra, ${ }^{4}$ J. T. Mitchell, ${ }^{7}$ G. Mitsuka, ${ }^{29,51}$ S. Miyasaka ${ }^{50,58}$ S. Mizuno, ${ }^{50,59}$ P. Montuenga, ${ }^{24}$ T. Moon,${ }^{63}$

D. P. Morrison, ${ }^{7}$ S. I. Morrow, ${ }^{60}$ T. Murakami,${ }^{32,50}$ J. Murata, ${ }^{50,52}$ K. Nagai,${ }^{58}$ K. Nagashima, ${ }^{21}$ T. Nagashima,${ }^{52}$ J. L. Nagle, ${ }^{12}$ M. I. Nagy, ${ }^{16}$ I. Nakagawa,${ }^{50,51}$ K. Nakano, ${ }^{50,58}$ C. Nattrass, ${ }^{57}$ T. Niida, ${ }^{59}$ R. Nouicer ${ }^{7,51}$ T. Novák, ${ }^{17,62}$ N. Novitzky, ${ }^{56}$ A. S. Nyanin, ${ }^{31}$ E. O’Brien, ${ }^{7}$ C. A. Ogilvie, ${ }^{27}$ J. D. Orjuela Koop, ${ }^{12}$ J. D. Osborn, ${ }^{39}$ A. Oskarsson, ${ }^{35}$ G. J. Ottino, ${ }^{43}$ K. Ozawa, ${ }^{29,59}$ V. Pantuev,${ }^{25}$ V. Papavassiliou, ${ }^{44}$ J. S. Park,${ }^{54}$ S. Park,${ }^{50,54,56}$ S. F. Pate, ${ }^{44}$ M. Patel, ${ }^{27}$ W. Peng, ${ }^{60}$ D. V. Perepelitsa, ${ }^{7,12}$ G. D. N. Perera, ${ }^{44}$ D. Yu. Peressounko, ${ }^{31}$ C. E. PerezLara, ${ }^{56}$ J. Perry, ${ }^{27}$ R. Petti, ${ }^{7}$ M. Phipps, ${ }^{7,24}$

C. Pinkenburg, ${ }^{7}$ R. P. Pisani, ${ }^{7}$ M. L. Purschke, ${ }^{7}$ P. V. Radzevich, ${ }^{53}$ K. F. Read ${ }^{46,57}$ D. Reynolds, ${ }^{55}$ V. Riabov, ${ }^{42,49}$

Y. Riabov, ${ }^{49,53}$ D. Richford, ${ }^{5}$ T. Rinn, ${ }^{27}$ S. D. Rolnick, ${ }^{8}$ M. Rosati, ${ }^{27}$ Z. Rowan, ${ }^{5}$ J. Runchey, ${ }^{27}$ A. S. Safonov, ${ }^{53}$

T. Sakaguchi, ${ }^{7}$ H. Sako, ${ }^{28}$ V. Samsonov, ${ }^{42,49}$ M. Sarsour, ${ }^{20}$ S. Sato ${ }^{28}$ B. Schaefer, ${ }^{60}$ B. K. Schmoll, ${ }^{57}$ K. Sedgwick, ${ }^{8}$ R. Seidl, ${ }^{50,51}$ A. Sen, ${ }^{27,57}$ R. Seto, ${ }^{8}$ A. Sexton, ${ }^{37}$ D. Sharma, ${ }^{56}$ I. Shein ${ }^{23}$ T.-A. Shibata, ${ }^{50,58}$ K. Shigaki, ${ }^{21}$ M. Shimomura, ${ }^{27,41}$ T. Shioya, ${ }^{59}$ P. Shukla, ${ }^{4}$ A. Sickles, ${ }^{24}$ C. L. Silva,${ }^{34}$ D. Silvermyr, ${ }^{35}$ B. K. Singh, ${ }^{3}$ C. P. Singh, ${ }^{3}$ V. Singh, ${ }^{3}$ M. J. Skoby,${ }^{39}$

M. Slunečka, ${ }^{9}$ M. Snowball, ${ }^{34}$ R. A. Soltz, ${ }^{33}$ W. E. Sondheim, ${ }^{34}$ S. P. Sorensen ${ }^{57}$ I. V. Sourikova, ${ }^{7}$ P. W. Stankus ${ }^{46}$ S. P. Stoll, ${ }^{7}$ T. Sugitate, ${ }^{21}$ A. Sukhanov, ${ }^{7}$ T. Sumita,${ }^{50}$ J. Sun,${ }^{56}$ Z. Sun,${ }^{15}$ J. Sziklai, ${ }^{62}$ K. Tanida,${ }^{28,51,54}$ M. J. Tannenbaum,

S. Tarafdar, ${ }^{60,61}$ A. Taranenko, ${ }^{42,55}$ G. Tarnai, ${ }^{15}$ R. Tieulent,${ }^{20,36}$ A. Timilsina, ${ }^{27}$ T. Todoroki, ${ }^{51,59}$ M. Tomášek, ${ }^{14}$

C. L. Towell, ${ }^{1}$ R. S. Towell, ${ }^{1}$ I. Tserruya ${ }^{61}$ Y. Ueda,${ }^{21}$ B. Ujvari,${ }^{15}$ H. W. van Hecke, ${ }^{34}$ J. Velkovska, ${ }^{60}$ M. Virius, ${ }^{14}$ V. Vrba, ${ }^{14,26}$ N. Vukman, ${ }^{64}$ X. R. Wang, ${ }^{44,51}$ Y. S. Watanabe, ${ }^{11}$ C. P. Wong, ${ }^{20}$ C. L. Woody ${ }^{7}$ C. Xu ${ }^{44}$ Q. Xu ${ }^{60}$ L. Xue, ${ }^{20}$ S. Yalcin, ${ }^{56}$ Y. L. Yamaguchi, ${ }^{51,56}$ H. Yamamoto, ${ }^{59}$ A. Yanovich, ${ }^{23}$ J. H. Yoo, ${ }^{30}$ I. Yoon, ${ }^{54}$ H. Yu, ${ }^{44,48}$ I. E. Yushmanov, ${ }^{31}$ W. A. Zajc, ${ }^{13}$ A. Zelenski, ${ }^{6}$ S. Zharko, ${ }^{53}$ and L. Zou ${ }^{8}$

(PHENIX Collaboration)

\author{
${ }^{1}$ Abilene Christian University, Abilene, Texas 79699, USA \\ ${ }^{2}$ Department of Physics, Augustana University, Sioux Falls, South Dakota 57197, USA \\ ${ }^{3}$ Department of Physics, Banaras Hindu University, Varanasi 221005, India \\ ${ }^{4}$ Bhabha Atomic Research Centre, Bombay 400 085, India \\ ${ }^{5}$ Baruch College, City University of New York, New York, New York 10010, USA \\ ${ }^{6}$ Collider-Accelerator Department, Brookhaven National Laboratory, Upton, New York 11973-5000, USA \\ ${ }^{7}$ Physics Department, Brookhaven National Laboratory, Upton, New York 11973-5000, USA \\ ${ }^{8}$ University of California-Riverside, Riverside, California 92521, USA \\ ${ }^{9}$ Charles University, Ovocný trh 5, Praha 1, 11636 Prague, Czech Republic \\ ${ }^{10}$ Chonbuk National University, Jeonju 561-756, Korea
}


${ }^{11}$ Center for Nuclear Study, Graduate School of Science, University of Tokyo, 7-3-1 Hongo, Bunkyo, Tokyo 113-0033, Japan

${ }^{12}$ University of Colorado, Boulder, Colorado 80309, USA

${ }^{13}$ Columbia University, New York, New York 10027 and Nevis Laboratories, Irvington, New York 10533, USA

${ }^{14}$ Czech Technical University, Zikova 4, 16636 Prague 6, Czech Republic

${ }^{15}$ Debrecen University, H-4010 Debrecen, Egyetem tér 1, Hungary

${ }^{16}$ ELTE, Eötvös Loránd University, H-1117 Budapest, Pázmány P. s. 1/A, Hungary

${ }^{17}$ Eszterházy Károly University, Károly Róbert Campus, H-3200 Gyöngyös, Mátrai út 36, Hungary

${ }^{18}$ Ewha Womans University, Seoul 120-750, Korea

${ }^{19}$ Florida State University, Tallahassee, Florida 32306, USA

${ }^{20}$ Georgia State University, Atlanta, Georgia 30303, USA

${ }^{21}$ Hiroshima University, Kagamiyama, Higashi-Hiroshima 739-8526, Japan

${ }^{22}$ Department of Physics and Astronomy, Howard University, Washington, DC 20059, USA

${ }^{23}$ IHEP Protvino, State Research Center of Russian Federation, Institute for High Energy Physics, Protvino 142281, Russia

${ }^{24}$ University of Illinois at Urbana-Champaign, Urbana, Illinois 61801, USA

${ }^{25}$ Institute for Nuclear Research of the Russian Academy of Sciences, prospekt 60-letiya Oktyabrya 7a, Moscow 117312, Russia

${ }^{26}$ Institute of Physics, Academy of Sciences of the Czech Republic,

Na Slovance 2, 18221 Prague 8, Czech Republic

${ }^{27}$ Iowa State University, Ames, Iowa 50011, USA

${ }^{28}$ Advanced Science Research Center, Japan Atomic Energy Agency,

2-4 Shirakata Shirane, Tokai-mura, Naka-gun, Ibaraki-ken 319-1195, Japan

${ }^{29}$ KEK, High Energy Accelerator Research Organization, Tsukuba, Ibaraki 305-0801, Japan

${ }^{30}$ Korea University, Seoul 136-701, Korea

${ }^{31}$ National Research Center “Kurchatov Institute," Moscow 123098, Russia

${ }^{32}$ Kyoto University, Kyoto 606-8502, Japan

${ }^{33}$ Lawrence Livermore National Laboratory, Livermore, California 94550, USA

${ }^{34}$ Los Alamos National Laboratory, Los Alamos, New Mexico 87545, USA

${ }^{35}$ Department of Physics, Lund University, Box 118, SE-221 00 Lund, Sweden

${ }^{36}$ IPNL, CNRS/IN2P3, Univ Lyon, Universit Lyon 1, F-69622 Villeurbanne, France

${ }^{37}$ University of Maryland, College Park, Maryland 20742, USA

${ }^{38}$ Department of Physics, University of Massachusetts, Amherst, Massachusetts 01003-9337, USA

${ }^{39}$ Department of Physics, University of Michigan, Ann Arbor, Michigan 48109-1040, USA

${ }^{40}$ Muhlenberg College, Allentown, Pennsylvania 18104-5586, USA

${ }^{41}$ Nara Women's University, Kita-uoya Nishi-machi Nara 630-8506, Japan

${ }^{42}$ National Research Nuclear University, MEPhI, Moscow Engineering Physics Institute, Moscow 115409, Russia

${ }^{43}$ University of New Mexico, Albuquerque, New Mexico 87131, USA

${ }^{44}$ New Mexico State University, Las Cruces, New Mexico 88003, USA

${ }^{45}$ Department of Physics and Astronomy, Ohio University, Athens, Ohio 45701, USA

${ }^{46}$ Oak Ridge National Laboratory, Oak Ridge, Tennessee 37831, USA

${ }^{47}$ IPN-Orsay, Univ. Paris-Sud, CNRS/IN2P3, Université Paris-Saclay, BP1, F-91406 Orsay, France

${ }^{48}$ Peking University, Beijing 100871, People's Republic of China

${ }^{49}$ PNPI, Petersburg Nuclear Physics Institute, Gatchina, Leningrad region 188300, Russia

${ }^{50}$ RIKEN Nishina Center for Accelerator-Based Science, Wako, Saitama 351-0198, Japan

${ }^{51}$ RIKEN BNL Research Center, Brookhaven National Laboratory, Upton, New York 11973-5000, USA

${ }^{52}$ Physics Department, Rikkyo University, 3-34-1 Nishi-Ikebukuro, Toshima, Tokyo 171-8501, Japan

${ }^{53}$ Saint Petersburg State Polytechnic University, St. Petersburg 195251, Russia

${ }^{54}$ Department of Physics and Astronomy, Seoul National University, Seoul 151-742, Korea

${ }^{55}$ Chemistry Department, Stony Brook University, SUNY, Stony Brook, New York 11794-3400, USA

${ }^{56}$ Department of Physics and Astronomy, Stony Brook University, SUNY, Stony Brook, New York 11794-3800, USA

${ }^{57}$ University of Tennessee, Knoxville, Tennessee 37996, USA

${ }^{58}$ Department of Physics, Tokyo Institute of Technology, Oh-okayama, Meguro, Tokyo 152-8551, Japan

${ }^{59}$ Tomonaga Center for the History of the Universe, University of Tsukuba, Tsukuba, Ibaraki 305, Japan

${ }^{60}$ Vanderbilt University, Nashville, Tennessee 37235, USA

${ }^{61}$ Weizmann Institute, Rehovot 76100, Israel 


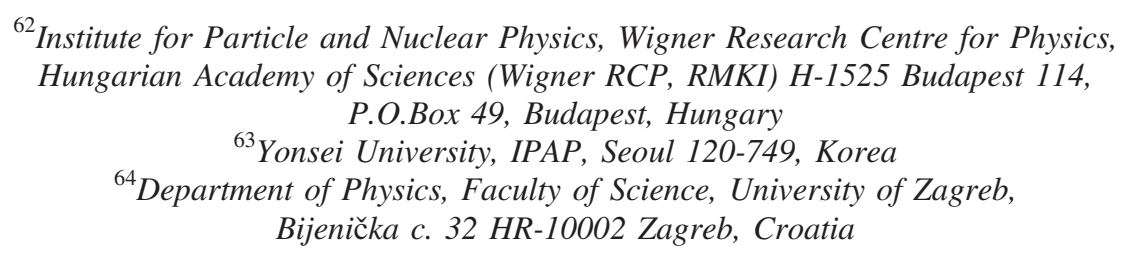

(Received 9 May 2018; published 17 October 2018)

\begin{abstract}
Dihadron and isolated direct photon-hadron angular correlations are measured in $p+p$ collisions at $\sqrt{s}=200 \mathrm{GeV}$. The correlations are sensitive to nonperturbative initial-state and final-state transverse momenta $k_{T}$ and $j_{T}$ in the azimuthal nearly back-to-back region $\Delta \phi \sim \pi$. To have sensitivity to small transverse momentum scales, nonperturbative momentum widths of $p_{\text {out }}$, the out-of-plane transversemomentum component perpendicular to the trigger particle, are measured. In this region, the evolution of $p_{\text {out }}$ can be studied when several different hard scales are measured. These widths are used to investigate possible effects from transverse-momentum-dependent factorization breaking. When accounting for the longitudinal-momentum fraction of the away-side hadron with respect to the near-side trigger particle, the widths are found to increase with the hard scale; this is qualitatively similar to the observed behavior in Drell-Yan and semi-inclusive deep-inelastic scattering interactions, where factorization is predicted to hold. The momentum widths are also studied as a function of center-of-mass energy by comparing to previous measurements at $\sqrt{s}=510 \mathrm{GeV}$. The nonperturbative jet widths also appear to increase with $\sqrt{s}$ at a similar $x_{T}$, which is qualitatively consistent to similar measurements in Drell-Yan interactions. Future detailed global comparisons between measurements of processes where transverse-momentum-dependent factorization is predicted to hold and be broken will provide further insight into the role of color in hadronic interactions.
\end{abstract}

DOI: $10.1103 /$ PhysRevD.98.072004

\section{INTRODUCTION}

QCD research has entered a period in which the focus of nucleon structure has shifted from a one-dimensional to a multidimensional picture. To take into account additional degrees of freedom besides the longitudinal momentum of partons within hadrons, the transverse-momentumdependent (TMD) framework, which also accounts for partons' transverse momentum, has been developed. Rather than parton distribution functions (PDFs) and fragmentation functions (FFs) being integrated over transversemomentum degrees of freedom, TMD PDFs (FFs) contain explicit dependence on the nonperturbative transverse momentum of the parton within the nucleon (hadron with respect to the fragmenting parton). This dependence offers a way to describe the three-dimensional momentum distribution of unpolarized partons within unpolarized hadrons, in addition to a variety of spin-spin and spinmomentum correlations when the parton and nucleon spin states are considered.

\footnotetext{
*Deceased.

${ }^{\dagger}$ PHENIX Spokesperson: akiba@rcf.rhic.bnl.gov
}

Published by the American Physical Society under the terms of the Creative Commons Attribution 4.0 International license. Further distribution of this work must maintain attribution to the author(s) and the published article's title, journal citation, and DOI. Funded by SCOAP ${ }^{3}$.
In the past decade, significant effort has been placed on measuring asymmetries that are sensitive to PDFs and FFs within the TMD framework. Semi-inclusive deep-inelastic scattering (SIDIS) and Drell-Yan (DY) measurements have shown empirical evidence for nonzero spin-momentum correlations in the initial state [1-5]. Additionally, $e^{+} e^{-}$ annihilation and SIDIS measurements have shown nonzero spin-momentum correlations in the final-state hadronization process [6-8]. With the development of robust and theoretically interpretable jet finding algorithms, there have also been several measurements studying nonperturbative hadronization in unpolarized $p+p$ collisions $[9,10]$ as well as spin-momentum correlations in polarized $p+p$ collisions $[11,12]$. Transverse single spin asymmetries of inclusive hadron production in $p+p$ collisions of up to $\sim 40 \%$ also indicate large spin-momentum correlations within the nucleon and/or the process of hadronization [13]; however, these measurements cannot probe functions within the TMD framework directly because there is not a simultaneous measurement of a small and hard transversemomentum scale.

The focus on multidimensional parton structure has brought the importance of soft gluon exchanges in hard interactions to the forefront of QCD research. In particular, the role of color exchanges due to soft gluon interactions with remnants of the hard scattering has brought to light fundamental predictions about QCD as a gauge-invariant 
quantum field theory. For example, the Sivers TMD PDF [14,15], which correlates the partonic transverse momentum with the nucleon spin, is predicted to exhibit modified universality when measured in the SIDIS and DY processes [16]. The underlying physical cause of this prediction is the interference between color fields in the two processes when an initial-state gluon is exchanged in DY vs a final-state gluon in SIDIS. The Sivers function has already been measured to be nonzero in SIDIS [1]. The first measurements of the DY-like $\mathrm{W}$ boson and DY transverse single spin asymmetry were recently reported $[17,18]$ and are consistent with the predicted sign change of the Sivers function. However, the uncertainties on the measurements are still large enough that a definitive conclusion cannot be drawn; more data will ultimately be necessary as the prediction is for the entire Sivers distribution as a function of the partonic longitudinal and transverse momentum $x$ and $k_{T}$.

In leading-order perturbative QCD processes where a colored parton is exchanged in the hard interaction, and thus color is necessarily present in both the initial and final states, soft gluon exchanges can lead to new effects in a TMD framework similarly to the predicted modified universality of certain TMD PDFs. In hadronic collisions where a final-state hadron is measured and the process is sensitive to a small transverse-momentum scale, factorization breaking has been predicted [19-22]. These processes can offer sensitivity to the non-Abelian nature of QCD. In processes where factorization is broken, the nonperturbative objects can no longer be factorized into a convolution of TMD PDFs and TMD FFs due to the complex color flows that are possible throughout the hard scattering and remnants of the collision and thus connect the initial- and final-state hadrons. In both cases of factorization breaking and modified universality of certain TMD PDFs, gluon exchanges with the remnants cannot be eliminated via gauge transformations. There have also been recent studies showing that factorization is broken for certain processes even at the collinear multiloop level [23,24]; however, the focus of this work is to search for TMD effects.

In the past decade, the role of color in hadronic interactions has also been explored in several different types of observables in $p+p$ collisions. The measurement of collective behavior in high-multiplicity $p+p$ collisions has prompted new studies of global observables in addition to interference effects between necessarily color connected multiple partonic interactions [25-27]. Measurements of dijet + jet and direct photon-jet + jet correlations at the Large Hadron Collider studying color-coherence effects have found effects from color-radiation patterns specific to hadronic collisions $[28,29]$. New dijet observables which rely on jet substructure have been shown to be sensitive to color flow in $t \bar{t}$ events [30]. With theoretical advances in jet substructure techniques, such as within soft-collinear effective theory [31], future measurements involving jet substructure may be an effective way to probe soft radiation patterns that allow quantification of color flow effects similarly to Ref. [30]. The advancement of both experimental and theoretical techniques has allowed the magnitude of effects from color flow and color connections to be probed in a variety of ways across different subfields of QCD.

A previous measurement in $p+p$ collisions at $\sqrt{s}=$ $510 \mathrm{GeV}$ found that nonperturbative momentum widths in dihadron and direct photon-hadron angular correlations binned in a fixed range of the away-side associated hadron $p_{T}$ decreased with the hard scale of the interaction, which is qualitatively opposite to what is expected from CollinsSoper-Sterman (CSS) evolution [32], which comes directly out of the derivation of TMD factorization [33]. This study is intended to extend the analysis of Ref. [32] at a smaller center-of-mass energy; therefore, this measurement supplants the previous PHENIX analysis at $\sqrt{s}=200 \mathrm{GeV}$ [34] with an approximately four times increase in integrated luminosity. The two different center-of-mass energies of Ref. [32] and this analysis allow TMD effects in dihadron and direct photon-hadron correlations to be studied as a function of $\sqrt{s}$.

\section{EXPERIMENT DETAILS}

In 2015, the PHENIX experiment collected data from $p+p$ collisions at $\sqrt{s}=200 \mathrm{GeV}$. A total minimum-bias integrated luminosity of $60 \mathrm{pb}^{-1}$ was used for the analysis of dihadron and direct photon-hadron angular correlations, from which data quality assurance and $z$ vertex cuts of $\left|z_{\mathrm{vtx}}\right|<30 \mathrm{~cm}$ were applied. The PHENIX detector measures two particle angular correlations via its electromagnetic calorimeter (EMCal) and drift chamber (DC) plus pad chamber (PC) tracking system located in two centralrapidity arms. The central arms are nearly back to back in azimuth, and each covers approximately $\Delta \phi \sim \pi / 2 \mathrm{rad}$ in the azimuthal angle and $\Delta \eta \sim 0.7$ units in pseudorapidity centered about midrapidity. A schematic diagram of the two central arms is shown in Fig. 1. Detailed descriptions of the PHENIX central arm spectrometers can be found in Refs. [35,36].

The PHENIX EMCal [36] comprises eight sectors, four in each arm. Six of the sectors are lead-scintillator sampling calorimeters, and the other two are lead-glass Čerenkov calorimeters. A high-energy-photon trigger in the EMCal is used to identify events with a high- $p_{T}$ photon. Photons are identified with a shower shape cut that removes charged hadrons as well as most high-energy clusters that overlap closely with another photon, which helps eliminate $\pi^{0}$ merging effects at high $p_{T}$. The granularity of the EMCal as well as the shower shape cuts allow for $\pi^{0}$ and $\eta$ reconstruction up to $\sim 20 \mathrm{GeV}$ in the diphoton channel, which allows for direct photon measurements as well. In this analysis, isolated photons are measured between $5<p_{T}<$ $15 \mathrm{GeV} / c$, and neutral pions are measured between $4<p_{T}<15 \mathrm{GeV} / c$. Previous $\pi^{0}, \eta$, and direct photon 


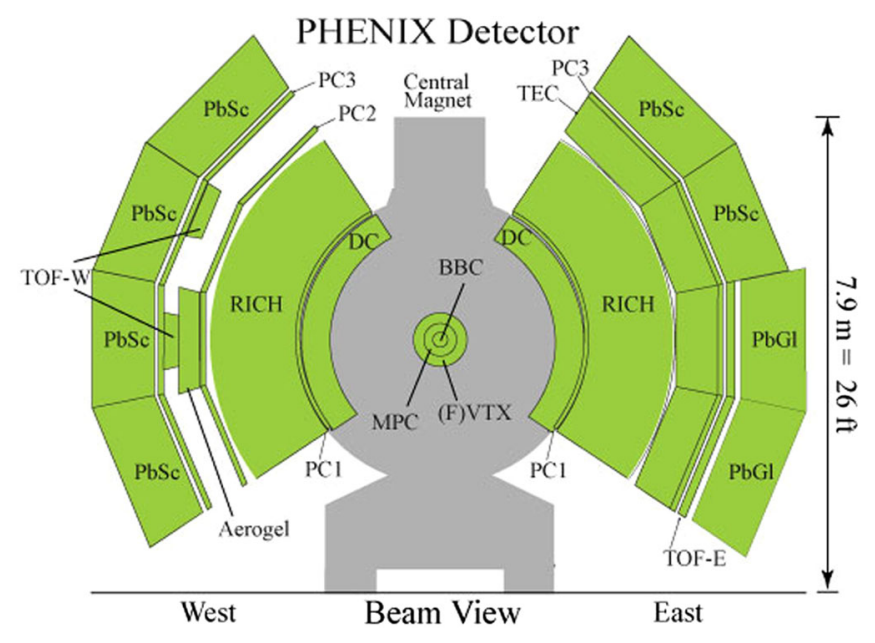

FIG. 1. A cross sectional view along the beam line shows the two central arms of the PHENIX detector.

cross sections as well as $\pi^{0}$-hadron or direct photon-hadron correlation can be found in Refs. [32,34,37-39].

The PHENIX tracking system [35] measures nonidentified charged hadrons with a DC and PC tracking system. The PC1, located radially behind the drift chamber, and PC3, located radially in front of the EMCal, allow for tracks identified in the DC to be matched with PC hits. The track matching condition in the PC1 and especially the PC3 reduces secondary tracks from conversions or decays. The ring-imaging Čerenkov system, located radially behind the DC in Fig. 1, is also used to remove electrons from the charged hadron sample. The tracking system is also used to suppress hadronic shower contamination when identifying photons by matching tracks in the DC and PC to showers in the EMCal and subsequently removing them. In this analysis, charged hadrons are collected over $0.5<$ $p_{T}<10 \mathrm{GeV} / c$. A previous nonidentified charged hadron cross section from PHENIX can be found in e.g., Ref. [40].

\section{ANALYSIS}

Dihadron and direct photon-hadron correlation functions are constructed following the methods of Refs. [32,34]. The correlation functions are constructed by measuring the raw number of correlated trigger particles and associated hadrons, where the trigger particle refers to either a leading $\pi^{0}$ or isolated photon. To account for the PHENIX acceptance, the yields are corrected by a mixed-event distribution by constructing correlations between trigger particles from one event and associated hadrons from a different event. The mixed event distribution is constructed on a run-by-run basis to quantify any changing efficiencies of the detector with time. The correlation functions are additionally corrected by a charged hadron efficiency to quantify the inefficiency of the DC and PC tracking system. The charged hadron efficiency is determined by simulating single particle hadrons in a GEANT3-based description of the PHENIX detector [41]. After corrections, the correlation functions are divided by the total number of trigger particles to construct a per-trigger yield. In this analysis, a 9\% uncertainty is assigned to the charged hadron efficiency, which amounts to an overall normalization uncertainty on the per-trigger yields. In total, the correlation functions correspond to yields within full azimuthal coverage and $|\eta|<0.35$. The correlations are constructed similarly to previous PHENIX analyses in Refs. [32,34,42-44].

\section{A. Statistical subtraction of decay photons}

The correlation functions can be constructed for any generic trigger-associated hadron combination. To identify direct photon-hadron correlations, an additional statistical subtraction must be applied to account for the background due to decay photon-hadron correlations. Reference [44] used a method which statistically subtracts the decay photon-hadron per-trigger yield component from a total inclusive photon-hadron per-trigger yield component with the following equation:

$$
Y_{\text {direct }}=\frac{1}{R_{\gamma}-1}\left(R_{\gamma} Y_{\text {inclusive }}-Y_{\text {decay }}\right)
$$

In this equation, $\mathrm{Y}$ is the per-trigger yield of a particular component denoted by the subscript, and $R_{\gamma}$ is the relative contribution of direct photons to decay photons defined as $R_{\gamma} \equiv N_{\text {inclusive }} / N_{\text {decay }}$. In Ref. [44], direct photons are defined as any photon not from a decay process and includes next-to-leading-order fragmentation photons.

To reduce the presence of fragmentation photons, Refs. [32,34] implemented an isolation cone criterion in the method, which we also use in this analysis. This has the added benefit of also reducing the decay photon background and thus providing a larger signal-to-background ratio for the direct photons. Additionally, photons that could be tagged as coming from $\pi^{0}$ or $\eta$ decays were removed. Because the tagging procedure does not remove all decay photons, Eq. (1) was modified to include these cuts and introduce isolated photon quantities,

$$
Y_{\text {direct }}^{\text {iso }}=\frac{1}{R_{\gamma}^{\text {iso }}-1}\left(R_{\gamma}^{\text {iso }} Y_{\text {inclusive }}^{\text {iso }}-Y_{\text {decay }}^{\text {iso }}\right)
$$

where again the trigger particles are noted in the subscripts for the various per-trigger yields and "iso" refers to both "isolated" and not tagged as coming from a $\pi^{0}$ decay. Here, $R_{\gamma}^{\text {iso }}$ is the relative contribution of isolated direct photons to isolated decay photons, defined in a way similar to $R_{\gamma}$ except with isolated quantities. While the subtraction procedure of Ref. [44] removes all decay photons, the modified isolated subtraction procedure removes background due to isolated decay photon-hadron correlations, which, in the PHENIX acceptance, are due most often to 
isolated neutral pion decays which decay asymmetrically such that the low- $p_{T}$ photon is not detected.

To implement the isolated statistical subtraction procedure, isolation and tagging cuts are applied at the eventby-event level in the data analysis. Inclusive photon candidates are removed from the analysis if a partner photon with $p_{T}>500 \mathrm{MeV} / c$ is found such that the invariant mass of the pair falls within the $\pi^{0}$ or $\eta$ invariant-mass regions, $0.118<m_{\gamma \gamma}<0.162$ and $0.5<$ $m_{\gamma \gamma}<0.6 \mathrm{GeV} / c^{2}$. In addition to the tagging cuts, an isolation cut is implemented. The isolation criterion is that the sum of EMCal energy and $p_{T}$ of charged tracks within a cone radius of 0.3 rad must be less than $10 \%$ of the candidate photons energy, similar to Ref. [34]. To reduce the effects of the detector acceptance, candidate isolated photons are also required to be $\sim 0.1 \mathrm{rad}$ from the edge of the detector in both $\phi$ and $\eta$. This forces a large portion of the cone to fall within the PHENIX acceptance.

The number of isolated decay photon-hadron pairs are not known a priori because an unknown fraction of the isolated photons still comes from decay processes. The isolated decay photon-hadron correlations are determined with a Monte Carlo generated probability density function that quantifies the probability of an isolated $\pi^{0}$ to decay to an isolated photon within the PHENIX acceptance such that the photon was not able to be tagged as coming from a decay process. The functions are used to map the isolated $\pi^{0}$-hadron correlations to the corresponding daughter isolated photon-hadron correlations for use in the statistical subtraction procedure, where the isolation criterion for the $\pi^{0}$ meson is the same as described above for single photons. In total, a 4\% systematic uncertainty was assigned for the statistical subtraction process, which includes additional background coming from higher mass state decays. With the probability functions, the decay photon-hadron pertrigger yields can be determined by

$$
Y_{\text {decay }}^{\text {iso }}=\frac{\sum_{N_{\pi-h}^{\text {iso }}} P\left(p_{T}^{\pi^{0}}, p_{T}^{\gamma}\right) N_{\pi^{0}-h}^{\text {iso }}}{\sum_{N_{\pi}^{\text {iso }}} P\left(p_{T}^{\pi^{0}}, p_{T}^{\gamma}\right) N_{\pi^{0}}^{\text {iso }}},
$$

where $P\left(p_{T}^{\pi^{0}}, p_{T}^{\gamma}\right)$ is the probability density function described above and $N$ is the number of isolated $\pi^{0}$-hadron pairs or isolated $\pi^{0}$ triggers measured as indicated in the subscripts.

To determine $R_{\gamma}^{\text {iso }}$, the quantity $R_{\gamma}$ must be corrected for the isolation and tagging cuts. The $R_{\gamma}$ was previously measured in Ref. [34], so these values are used and corrected with the measured isolation and tagging efficiencies of this analysis. The quantity is the ratio of isolated inclusive photons to isolated decay photons, is dependent only on the photon $p_{T}$, and can be written as

$$
\begin{aligned}
R_{\gamma}^{\text {iso }}\left(p_{T}^{\gamma}\right)= & \frac{N_{\text {inclusive }}^{\text {iso }}}{N_{\text {decay }}^{\text {iso }}} \\
= & \frac{R_{\gamma}}{\left(1-\epsilon_{\text {decay }}^{\text {tag }}\right)\left(1-\epsilon_{\text {decay }}^{\text {niso }}\right)} \\
& \times \frac{N_{\text {inclusive }}-N_{\text {decay }}^{\text {tag }}-N_{\text {inclusive }}^{\text {niso }}}{N_{\text {inclusive }}},
\end{aligned}
$$

where "niso" refers to "not isolated," $\epsilon_{\text {decay }}^{\text {tag }}$ is the tagging efficiency, $\epsilon_{\text {decay }}^{\text {niso }}$ is the isolation efficiency, and the various $N$ values are the number of photons that correspond to the subscript and superscript with which they are associated. The bottom-most fraction in Eq. (4) is simply the number of isolated inclusive photons divided by the total number of inclusive photons and can be determined by just counting the photons that pass the necessary cuts. The decay photon tagging efficiency can be written as $\epsilon_{\text {decay }}^{\text {tag }}=R_{\gamma} N_{\text {decay }}^{\text {tag }} / N_{\text {inclusive }}$ and can also be determined by counting the number of photons that pass the cuts. To determine the isolation efficiency, the isolation cut is applied at the level of the parent $\pi^{0}$, and the decay probability functions are used to map the effect to the daughter photons. The isolation efficiency can be written as

$$
\epsilon_{\text {decay }}^{\text {niso }}=\left(1+\frac{\sum_{\pi} P\left(p_{T}^{\pi^{0}}, p_{T}^{\gamma}\right) \cdot N_{\pi}^{\text {iso }}}{\sum_{\pi} P\left(p_{T}^{\pi^{0}}, p_{T}^{\gamma}\right) \cdot N_{\pi}^{\text {niso }}}\right)^{-1} .
$$

Therefore, all of the necessary components can be determined to calculate $R_{\gamma}^{\text {iso }}$. Figure 2 shows the measured values of $R_{\gamma}^{\text {iso }}$ as a function of $p_{T}^{\gamma}$ in this analysis compared to the previous PHENIX publication [34] at the same $\sqrt{s}$. The values show consistency with the previous analysis and are all larger than unity, indicating the signal-tobackground ratio of the isolated direct photons to decay

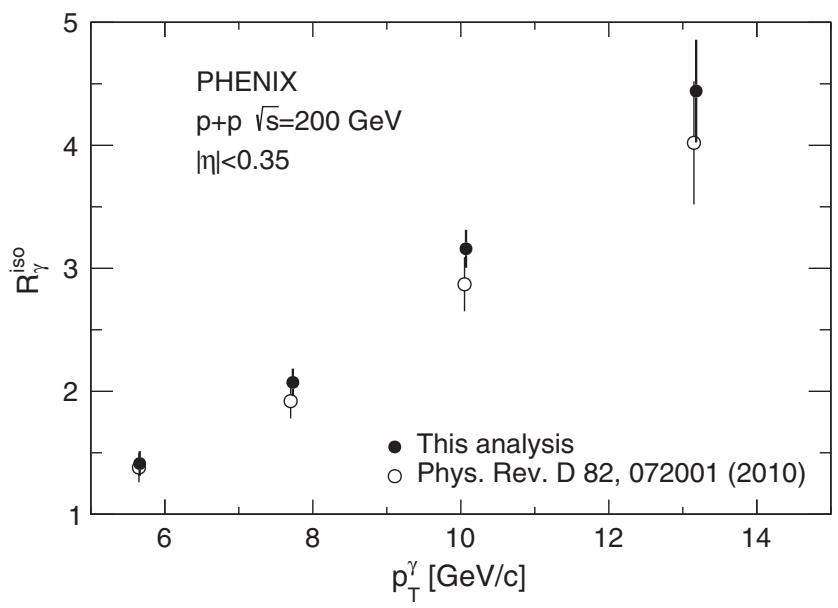

FIG. 2. Measured $R_{\gamma}^{\text {iso }}$ in this analysis compared to Ref. [34]. The statistic and systematic uncertainties are added in quadrature and are dominated by the systematic uncertainties. 
photons. These can also be compared to the values of $R_{\gamma}$ in Refs. $[34,44]$, showing that the tagging and isolation cuts increase the signal-to-background ratio.

\section{RESULTS}

\section{A. Azimuthal correlations}

Figure 3 shows the correlation functions measured in various $p_{T}^{\text {trig }}$ and $p_{T}^{\text {assoc }}$ bins. In the figure, the red open points are the dihadron correlations, while the filled blue points are the isolated direct photon-hadron correlations. A black dashed line estimates the underlying event pedestal to emphasize the jet yields. The per-trigger yields as a function of $\Delta \phi$ show the expected visual features of dihadron and direct photon-hadron correlations. The dihadron correlations have two peaks at $\Delta \phi \sim 0$ and $\pi$ corresponding to intrajet and back-to-back jet correlations, respectively. The isolated direct photon-hadron correlations show away-side yields that are consistently smaller than the corresponding $\pi^{0}$-hadron yields, which is to be expected because the $\pi^{0}$-hadron correlations probe larger hard scales due to the $\pi^{0}$ being a fragment of the jet. Note
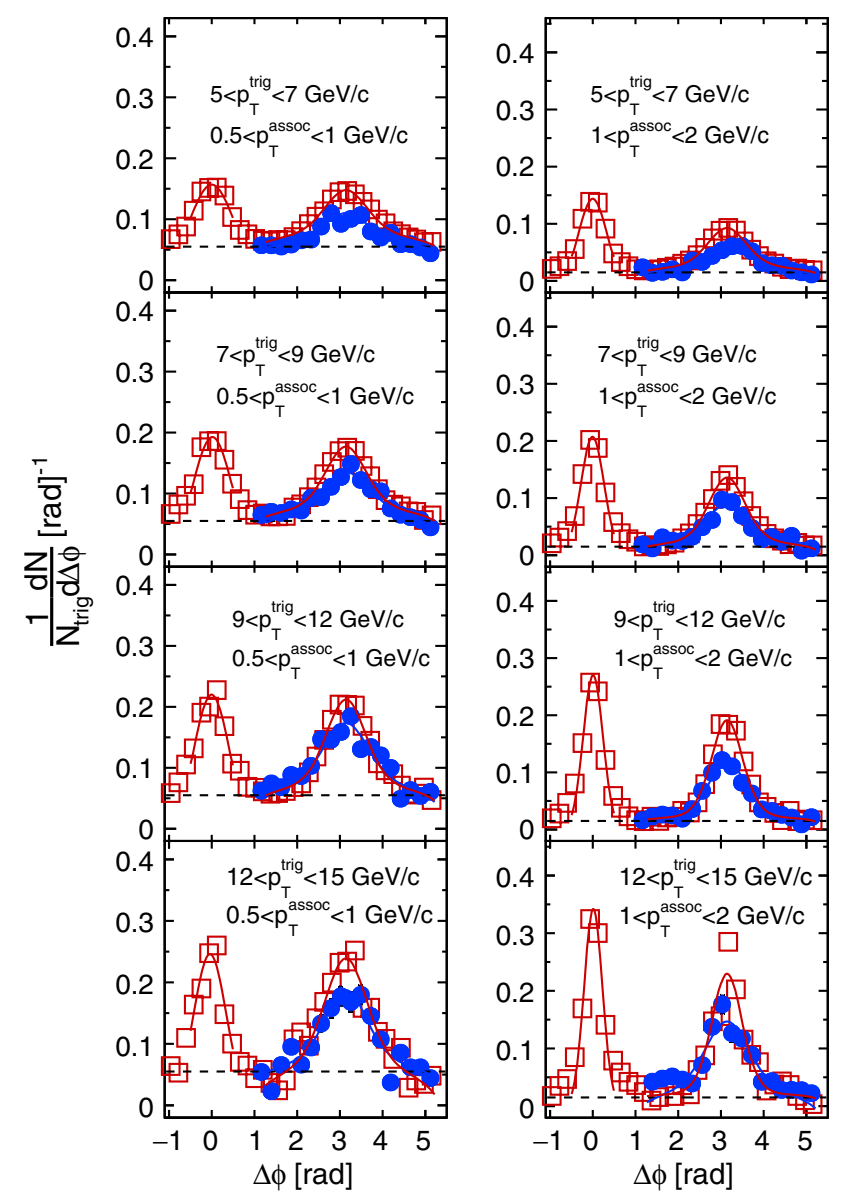

that a $9 \%$ charged hadron normalization uncertainty is not explicitly shown on any of figures displaying the pertrigger yields; this is denoted in each of the captions where the uncertainty applies.

\section{B. $p_{\text {out }}$ distributions}

The hard scattering quantity $p_{\text {out }}=p_{T}^{\text {assoc }} \sin \Delta \phi$, the transverse momentum component of the associated hadron with respect to the trigger particle axis, is defined kinematically in Fig. 4. Rather than constructing the per-trigger yields as a function of $\Delta \phi$, they are instead constructed as a function of $p_{\text {out }}$ in a way similar to the $\Delta \phi$ correlations. These distributions are the quantity of interest because the nonperturbative TMD structure can be observed from the correlation functions; additionally, they have the advantage that the nonperturbative component of the away-side jet width can be separated from the perturbative component in momentum space with a transition region between the two components.

Reference [32] found that the nonperturbative momentum widths of the $p_{\text {out }}$ distributions binned in a fixed range of $p_{T}^{\text {assoc }}$ decreased with the hard scale $p_{T}^{\text {trig }}$, contrary to
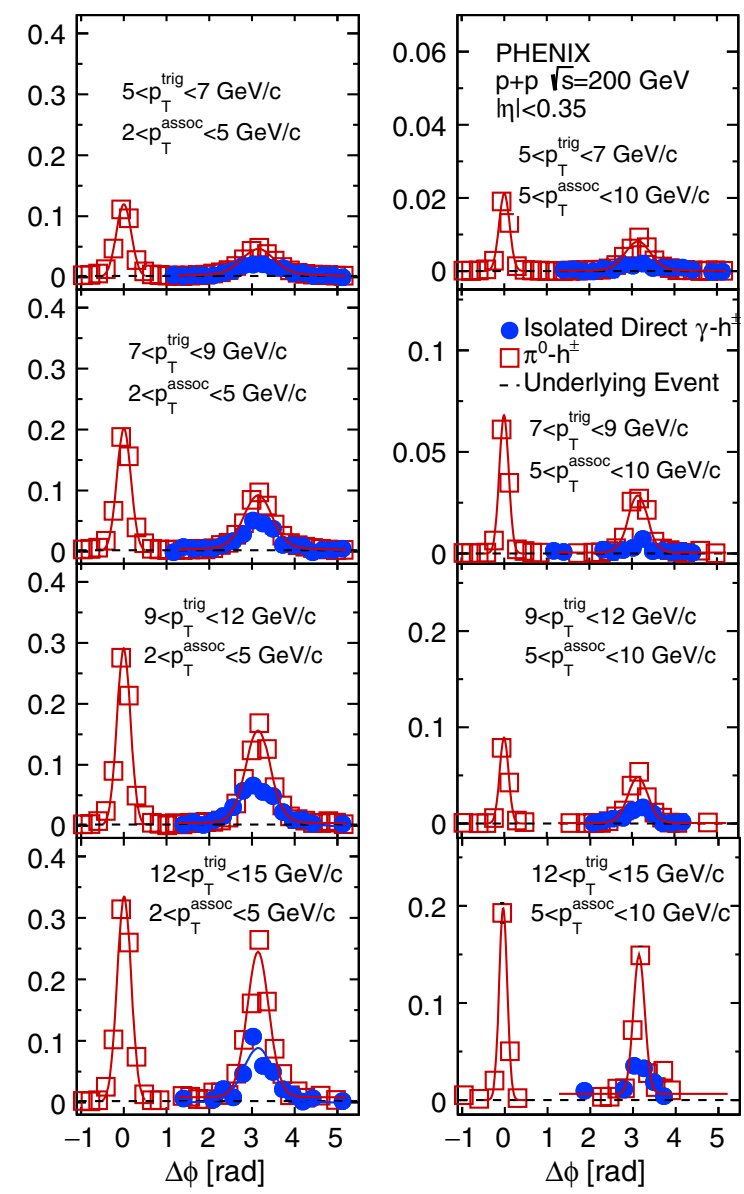

FIG. 3. The per-trigger yields are shown as a function of $\Delta \phi$ in several $p_{T}^{\text {trig }} \otimes p_{T}^{\text {assoc }}$ bins. The black dashed line shows an estimation of the underlying event yield to more clearly show the away-side jet yield. The $9 \%$ charged hadron normalization uncertainty is not explicitly shown on the figure. 
(a)

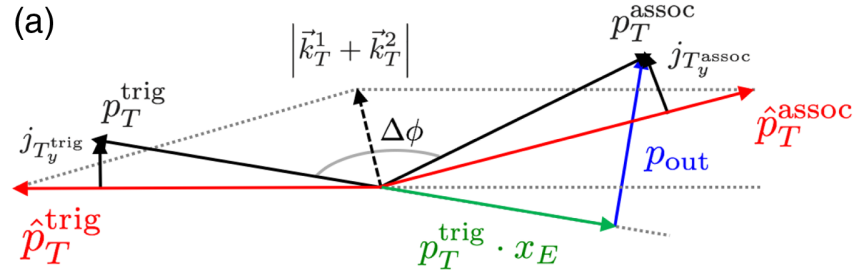

(b)

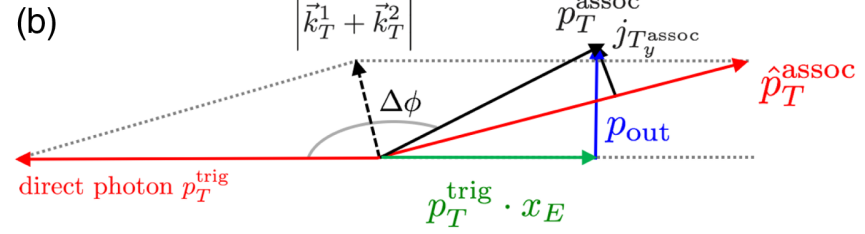

FIG. 4. A diagram which shows the hard scattering kinematics of a nearly back-to-back correlation for (a) dihadron and (b) direct photon-hadron events, adapted from Ref. [32]. Two hard-scattered partons, shown in red, are acoplanar due to the initial-state $\vec{k}_{T}^{1}$ and $\vec{k}_{T}^{2}$ of the colliding partons. The partons result in a trigger and associated jet fragment $p_{T}^{\text {trig }}$ and $p_{T}^{\text {assoc }}$ with a transversemomentum component perpendicular to the jet axis $j_{T_{y}^{\text {trig }}}$ and $j_{T_{y}^{a s s o c}}$ in the transverse plane, which are assumed to be Gaussian such that $\sqrt{\left\langle j_{T}^{2}\right\rangle}=\sqrt{2\left\langle j_{T_{y}^{\text {tig }}}^{2}\right\rangle}=\sqrt{2\left\langle j_{T_{y}^{\text {assoc }}}^{2}\right\rangle}$. For direct photons (b) $p_{T}^{\text {trig }}$ corresponds to the hard scattering vector because the direct photon is produced from the hard scattering. In each figure, the quantity $x_{E}$ is labeled as the green vector and approximates the momentum fraction $z$ of the final-state away-side hadron.

what is qualitatively expected from CSS evolution. The $p_{\text {out }}$ distributions were binned in a fixed $p_{T}^{\text {assoc }}$ range only, which means that the longitudinal-momentum fraction $z$ of the away-side hadron with respect to the away-side jet was generally decreasing as the hard scale increased. Here, we bin the $p_{\text {out }}$ correlation distributions as a function of the quantity $x_{E}$, which is defined in Ref. [34] as

$$
x_{E} \equiv-\frac{p_{T}^{\text {trig }} \cdot p_{T}^{\text {assoc }}}{\left|p_{T}^{\text {trig }}\right|^{2}}=-\frac{\left|p_{T}^{\text {assoc }}\right|}{\left|p_{T}^{\text {trig }}\right|} \cos \Delta \phi
$$

and is geometrically shown in Fig. 4. Because full jet reconstruction within PHENIX severely limits available statistics due to the limited acceptance, this quantity can be used as a proxy for $z$, the longitudinal-momentum fraction of the associated away-side hadron with respect to the away-side jet. Although $x_{E}$ is an approximation for $z$, this alternative binning allows for a clearer comparison of the $p_{\text {out }}$ distributions between hard scales as the associated hadrons are then binned in a similar kinematic way that is normalized by the near-side $p_{T}^{\text {trig }}$.

Because $x_{E}$ is only a proxy for $z$, there is an embedded dependence on the correlation between these two quantities for both the $\pi^{0}$-hadron and direct photon-hadron correlations. Previous correlations measurements from PHENIX have shown that the quantity $\hat{x}_{h}=\hat{p}_{T}^{\text {assoc }} / \hat{p}_{T}^{\text {trig }}$, where quantities with a hat indicate partonic quantities, is on average less than unity [34], and this has also been shown in direct photon-jet correlations [45]. Thus, for direct-photon hadron correlations, $z>x_{E}$. For $\pi^{0}$-hadron correlations, there is an additional dependence on $\left\langle z_{T}\right\rangle=$ $p_{T}^{\text {trig }} / \hat{p}_{T}^{\text {trig }}$, which is roughly 0.6 at Relativistic Heavy Ion Collider (RHIC) energies [43]. Thus, for $\pi^{0}$-hadron correlations, on average $z<x_{E}$; therefore, the dihadron and direct photon-hadron correlations are on average probing different values of the away-side hadron momentum fraction $z$.

The $p_{\text {out }}$ distributions for $p+p$ collisions at $\sqrt{s}=$ $200 \mathrm{GeV}$ binned in $0.1<x_{E}<0.5$ are shown in Fig. 5 . The open points are the $\pi^{0}$-hadron correlations, while the filled points are the direct photon-hadron correlations. In constructing the correlations, the underlying event was statistically subtracted following a method similar to Ref. [32]. The functions used to statistically subtract the underlying event are shown as fits to the away-side distributions in Fig. 3. Although the correlation functions are binned in $x_{E}$ instead of $p_{T}^{\text {assoc }}$, there is still a clear transition from nonperturbative to perturbative sensitivity in the distributions. This is highlighted by the Gaussian fits to the small $p_{\text {out }}$ region $[-1.1,1.1] \mathrm{GeV} / c$ in the figure, where the fits clearly fail at describing the correlations at large $p_{\text {out }}$. We also note that Ref. [32] found that the large $p_{\text {out }}$ region was described reasonably well with a Kaplan fit; here, the distributions are not described by a Kaplan fit due to the smaller center-of-mass energy. When $\sqrt{s}$ is smaller, it is less likely that a high $p_{T}$ gluon radiation will occur such that $p_{\text {out }}$ is large. This causes the $p_{\text {out }}$ distributions to fall more quickly toward zero at large $p_{\text {out }}$.

The widths of the Gaussian fits are extracted to quantify the evolution of the nonperturbative away-side jet widths as a function of $p_{T}^{\text {trig }}$. Systematic uncertainties are evaluated

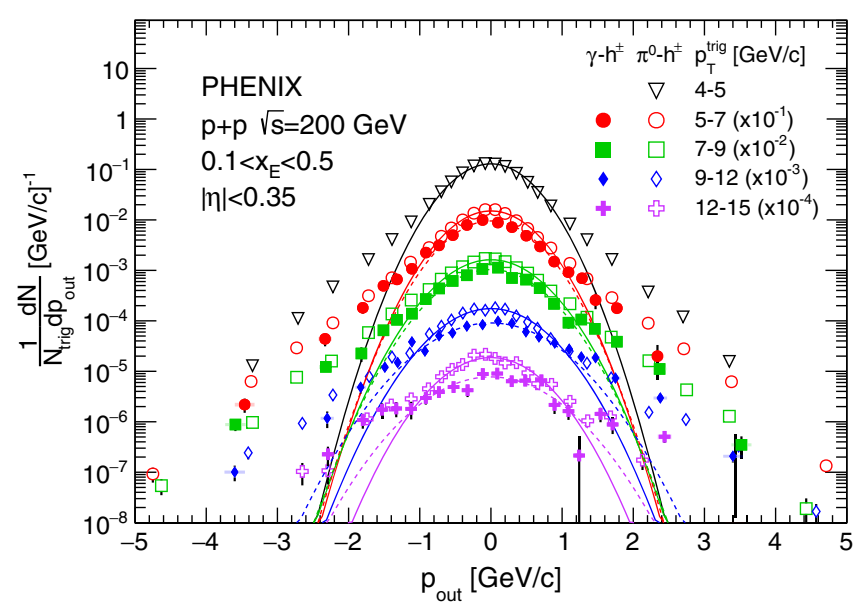

FIG. 5. The $p_{\text {out }}$ distributions are shown for dihadron and direct photon-hadron correlations, binned in $x_{E}$. The $9 \%$ charged hadron normalization uncertainty is not explicitly shown on the figure. 


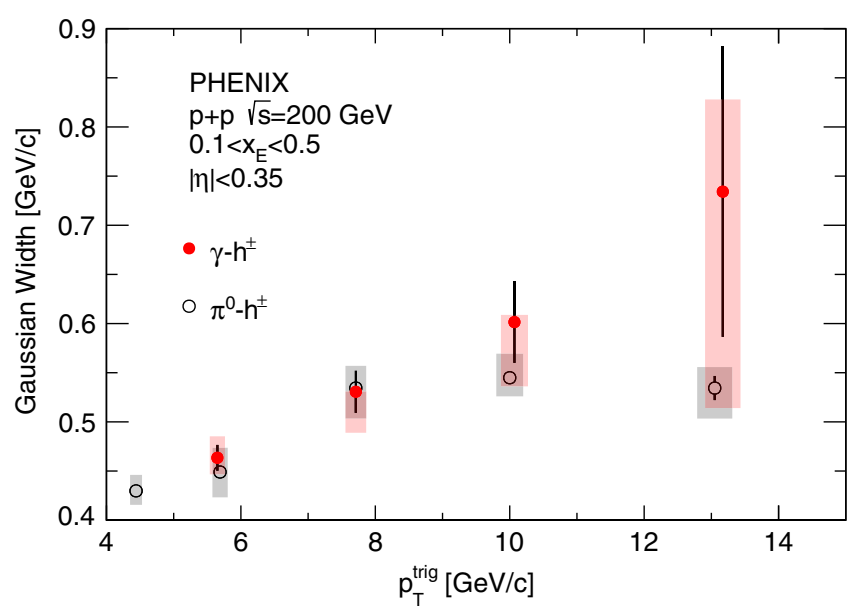

FIG. 6. The nonperturbative away-side jet widths as a function of $p_{T}^{\text {trig }}$ at $\sqrt{s}=200 \mathrm{GeV}$ for both direct photon-hadron and dihadron correlations.

by adjusting the fit region by $\pm 0.2 \mathrm{GeV} / c$ and taking the absolute difference of the resulting Gaussian width. The values are shown in Fig. 6 and Table I and clearly demonstrate that the widths increase with $p_{T}^{\text {trig }}$. This is in contrast to Ref. [32], where the widths decreased as a function of $p_{T}^{\text {trig }}$ when the $p_{\text {out }}$ distributions were binned in a fixed $p_{T}^{\text {assoc }}$ range and thus not in a way to account for the longitudinal-momentum fraction of the away-side hadron with respect to the near-side trigger particle.

To study the dependence of the nonperturbative momentum widths on the fragmentation quantity $x_{E}$, the $p_{\text {out }}$ distributions were constructed as a function of $x_{E}$. To have sufficient statistical precision, and to compare to the $\sqrt{s}=510 \mathrm{GeV}$ data which are described later, the distributions were integrated over a larger range of $7<$ $p_{T}^{\text {trig }}<12 \mathrm{GeV} / c$. The $p_{\text {out }}$ distributions are shown in Fig. 7 , and the nonperturbative structure is fit with a

TABLE I. Gaussian widths of $p_{\text {out }}$ for direct photon-hadron and dihadron correlations in $p+p$ collisions at $\sqrt{s}=200 \mathrm{GeV}$. Units are $\mathrm{GeV} / c$ for both $\left\langle p_{T}^{\text {trig }}\right\rangle$ and the Gaussian widths.

\begin{tabular}{lcccc}
\hline \hline Trigger type & $\left\langle p_{T}^{\text {trig }}\right\rangle$ & Gaussian width & Statistical & Systematic \\
\hline$\pi^{0}$ & 4.44 & 0.429 & 0.001 & ${ }_{-0.014}^{+0.016}$ \\
& 5.69 & 0.449 & 0.001 & ${ }_{-0.026}^{+0.024}$ \\
& 7.71 & 0.534 & 0.002 & ${ }_{-0.022}^{+0.022}$ \\
& 10.1 & 0.545 & 0.004 & ${ }_{-0.018}^{+0.024}$ \\
& 13.1 & 0.534 & 0.012 & ${ }_{-0.031}^{+0.021}$ \\
Direct photon & 5.65 & 0.46 & 0.01 & ${ }_{-0.02}^{+0.02}$ \\
& 7.71 & 0.53 & 0.02 & ${ }_{-0.04}^{+0.01}$ \\
& 10.1 & 0.60 & 0.04 & ${ }_{-0.06}^{+0.01}$ \\
& 13.2 & 0.73 & 0.15 & ${ }_{-0.02}^{+0.09}$ \\
\hline \hline
\end{tabular}

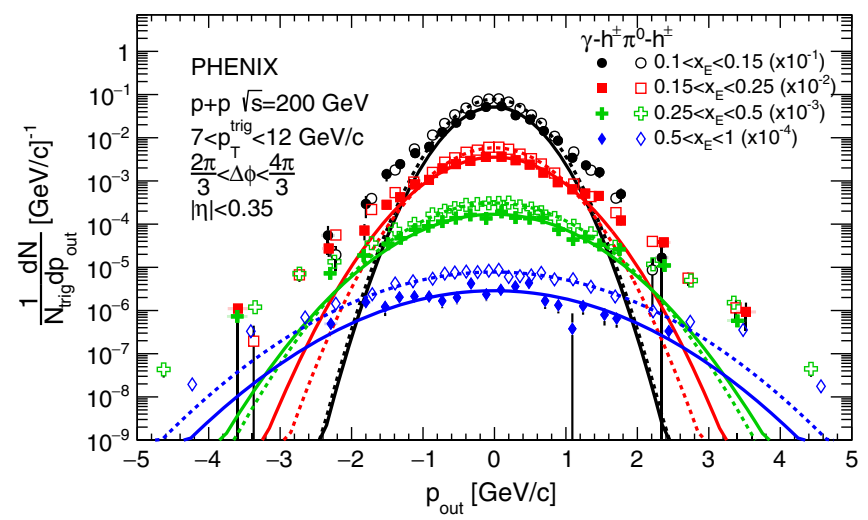

FIG. 7. The $p_{\text {out }}$ distributions are shown in several bins of $x_{E}$, integrated over a range of $p_{T}^{\text {trig }}$. The $9 \%$ charged hadron normalization uncertainty is not explicitly shown on the figure.

Gaussian function, shown as dashed lines and solid lines for $\pi^{0}$ and direct photon triggered correlations, respectively. The nonperturbative to perturbative transition is still visible; however, the region of both large $p_{\text {out }}$ and $x_{E}$ lacks statistical precision. The Gaussian fits are performed in varying regions of $p_{\text {out }}$, depending on the $x_{E}$ bin because the nonperturbative structure strongly depends on the $x_{E}$ bin probed. The Gaussian widths are extracted and shown as a function of $x_{E}$ in Fig. 8, where the systematic uncertainties on the widths are estimated in a way similar to the previous nonperturbative momentum widths.

To compare the results measured here to the previous PHENIX data at $\sqrt{s}=510 \mathrm{GeV}$, the data from Ref. [32] were rebinned in $x_{E}$ similarly to the results shown here. The nonperturbative momentum widths are extracted from Gaussian fits to the small $p_{\text {out }}$ region, and the widths from both center-of-mass energies are shown as a function of $p_{T}^{\text {trig }}$ in Fig. 9 and in Table II. Note that only the $\pi^{0}$-hadron

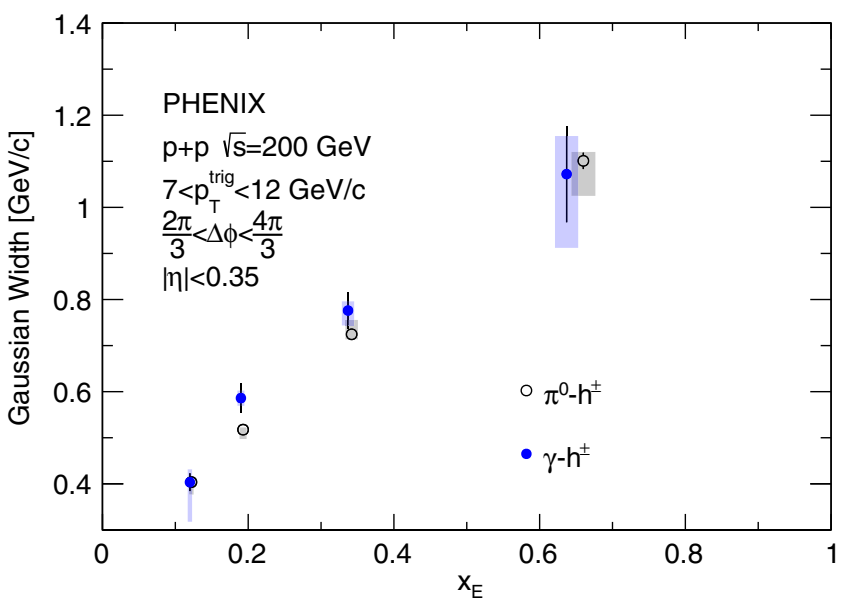

FIG. 8. The Gaussian widths of $p_{\text {out }}$ as a function of $x_{E}$ are shown for both $\pi^{0}$ and direct photon triggered correlations. 


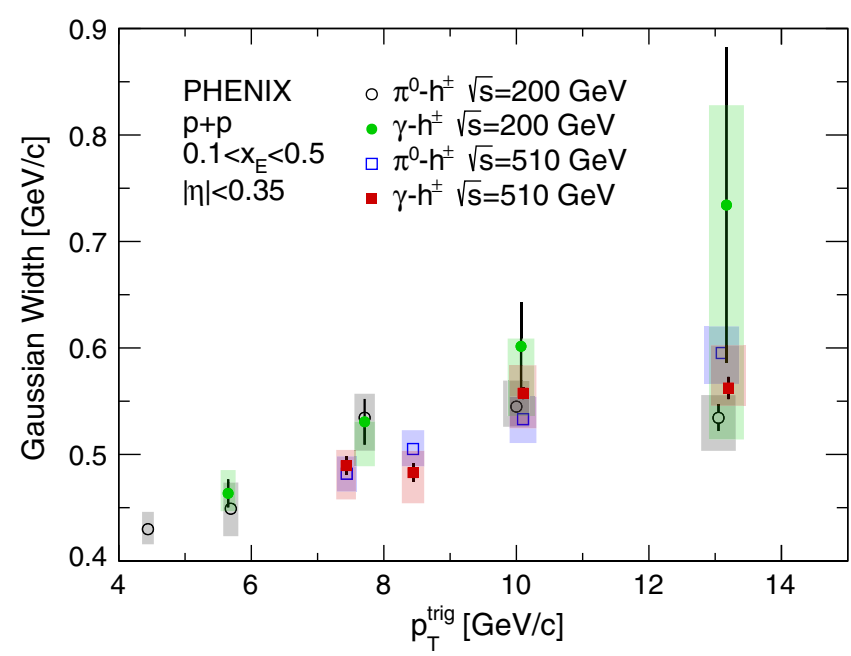

FIG. 9. The Gaussian widths extracted from the $p_{\text {out }}$ distributions in both $\sqrt{s}=200 \mathrm{GeV}$ and $\sqrt{s}=510 \mathrm{GeV}$ are shown as a function of $p_{T}^{\text {trig }}$.

correlations with $p_{T}^{\text {trig }}>7 \mathrm{GeV} / c$ were reanalyzed; this is because, due to detector capabilities, there was a minimum $p_{T}^{\text {assoc }}$ limit of $0.7 \mathrm{GeV} / c$ in the $\sqrt{s}=510 \mathrm{GeV}$ analysis [32]. For the minimum $x_{E}$ cut of 0.1 to be unbiased, we require that the $p_{T}^{\text {trig }}>7 \mathrm{GeV} / c$ in the $\sqrt{s}=510 \mathrm{GeV}$ data. The values at the two different center-of-mass energies are consistent with one another at a similar $p_{T}^{\text {trig }}$ within uncertainties. Figure 10 shows the nonperturbative Gaussian widths as a function of $x_{E}$ at the two different center-of-mass energies. The nonperturbative Gaussian widths also show little dependence as a function of $x_{E}$ on the center-of-mass energy. The data show that, at the hard scales and energies probed by RHIC, the nonperturbative away-side widths are consistent within uncertainties at two different center-of-mass energies. Similar conclusions have been drawn by the STAR Collaboration, where polarized TMD observables are consistent within uncertainties between $\sqrt{s}=200$ and $500 \mathrm{GeV}[11,12]$.

TABLE II. Gaussian widths of $p_{\text {out }}$ for direct photon-hadron and dihadron correlations in $p+p$ collisions at $\sqrt{s}=510 \mathrm{GeV}$ in a fixed $x_{E}$ bin, as seen in Fig. 11 and rebinned from Ref. [32]. Units are $\mathrm{GeV} / c$ for both $\left\langle p_{T}^{\text {trig }}\right\rangle$ and the Gaussian widths.

\begin{tabular}{lcccc}
\hline \hline Trigger type & $\left\langle p_{T}^{\text {trig }}\right\rangle$ & Gaussian width & Stat. & Sys. \\
\hline$\pi^{0}$ & 7.44 & 0.482 & 0.001 & ${ }_{-0.017}^{+0.016}$ \\
& 8.44 & 0.505 & 0.001 & ${ }_{-0.016}^{+0.018}$ \\
& 10.1 & 0.533 & 0.001 & ${ }_{-0.022}^{+0.021}$ \\
& 13.1 & 0.595 & 0.001 & ${ }_{-0.029}^{+0.025}$ \\
Direct photon & 7.43 & 0.489 & 0.009 & ${ }_{-0.032}^{+0.015}$ \\
& 8.44 & 0.483 & 0.009 & ${ }_{-0.029}^{+0.021}$ \\
& 10.1 & 0.558 & 0.007 & ${ }_{-0.033}^{+0.026}$ \\
& 13.2 & 0.562 & 0.011 & ${ }_{-0.017}^{+0.040}$ \\
\hline \hline
\end{tabular}

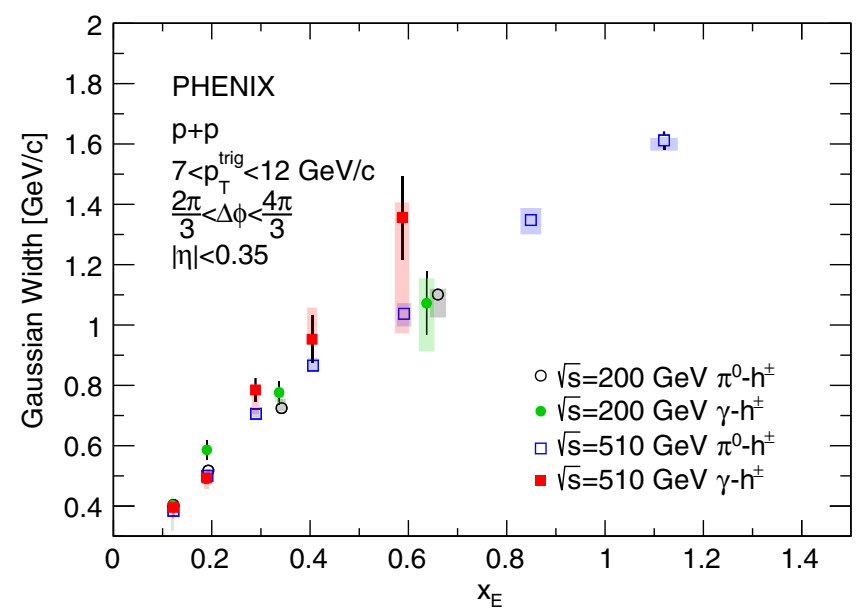

FIG. 10. The nonperturbative Gaussian widths are shown as a function of $x_{E}$ at two different center-of-mass energies.

The nonperturbative away-side jet widths are also shown as a function of $x_{T}=2 p_{T}^{\text {trig }} / \sqrt{s}$ in Fig. 11. The Gaussian widths do not appear to scale with $x_{T}$; however, they appear to show qualitatively similar behavior to DY interactions. The nonperturbative momentum widths sensitive to a small transverse-momentum scale increase with $\sqrt{s}$ at a similar $x_{T}$. This behavior can be observed in TMD momentum widths measured from DY data as a function of $\sqrt{s}$ and $\sqrt{\tau}=x_{1} x_{2}$, where $x_{1}$ and $x_{2}$ are the partonic momentum fractions of the quark-antiquark pair (see e.g., Ref. [46]). However, it is interesting to note that in DY at similar $M_{\mu \mu}$ nonperturbative momentum widths clearly rise with $\sqrt{s}$, while in the measurements presented here, as well as other polarized TMD observables from RHIC [11,12], nonperturbative momentum widths are consistent with each other as a function of $p_{T}^{\text {trig }}$ and $\sqrt{s}$.

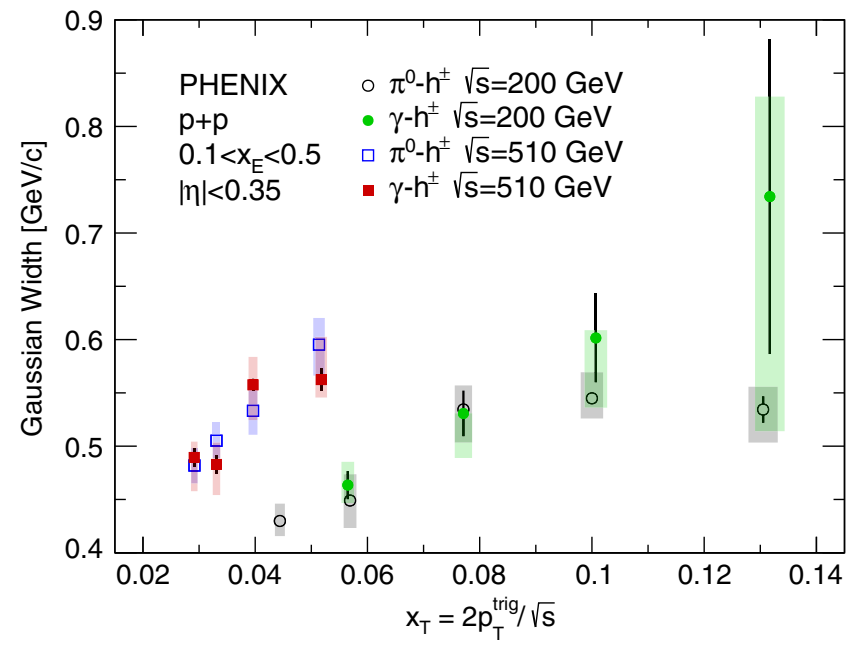

FIG. 11. The Gaussian widths extracted from the $p_{\text {out }}$ distributions in both $\sqrt{s}=200 \mathrm{GeV}$ and $\sqrt{s}=510 \mathrm{GeV}$ are shown as a function of $x_{T}=2 p_{T}^{\text {trig }} / \sqrt{s}$. 


\section{CONCLUSIONS}

Dihadron and direct photon-hadron correlations were measured at the PHENIX experiment in $p+p$ collisions at $\sqrt{s}=200 \mathrm{GeV}$. These processes have been predicted to violate factorization in a TMD framework due to soft gluon exchanges with remnants that are possible in both the initial and final states in hadronic collisions [21,22]. To better interpret the effects that were studied in Ref. [32], the $p_{\text {out }}$ distributions were binned in the hard scattering variable $x_{E}$ to provide more control over the fragmentation dependence as a function of the hard scale of the interaction. When accounting for the away-side hadron $p_{T}^{\text {assoc }}$ with respect to the near-side $p_{T}^{\text {trig }}$, the nonperturbative momentum widths are found to increase with the hard scale of the interaction $p_{T}^{\text {trig }}$. This is qualitatively similar to DY interactions in that nonperturbative momentum widths sensitive to a small transverse-momentum scale increase with the hard scale of the interaction. This behavior has been verified phenomenologically in both DY and SIDIS processes [47-51], and this measurement shows that this behavior is similar in processes which are predicted to break factorization.

The idea of quantum-correlated partons across colliding hadrons due to color flow, predicted from rigorous TMD theory, represents a novel way of considering hadronic interactions. The motivation of the present study was to search for obvious differences in these types of processes compared to ones where color can only be exchanged in either the initial or final state. For the time being, without observation of qualitative differences, the only obvious way to quantify factorization-breaking effects will be to compare data in processes such as those presented here to calculations assuming factorization holds. Such calculations are not available at this time, largely due to the fact that, even for unpolarized observables, the phenomenology of TMD functions is not as advanced as that of collinear functions. For example, significant theoretical focus has recently been placed on matching the TMD and collinear frameworks [52,53], understanding TMD evolution [54], or incorporating more data into global fits. The first global fit of TMD data was very recently reported [55], and in particular, the study calls for more data covering a broader range of kinematic variables to constrain future global fits. Additionally, TMD-evolution predictions still vary significantly depending on certain nonperturbative inputs [54]. Future comparisons of magnitudes, shapes, and evolution of these observables to calculations should be made to better understand effects from complex color flows.

To study the properties of non-Abelian quantum field theories, experiments should perform further measurements sensitive to color correlations. For example, spin asymmetries in the direct photon-jet channel [56] or in polarized $p+A$ collisions [57] have been predicted to arise due to factorization-breaking effects. Additional unpolarized processes may also provide input; e.g., nearly back-to-back direct photon-quarkonium production could be used to study the color dependence of observables, depending on whether or not the quarkonium state is produced in a color singlet or color octet state. The exploration of the role of color in hadronic interactions is deeply connected to the unique properties of QCD, and future analyses will explore and constrain the magnitudes of these effects.

It is unfortunate that theoretical calculations are not available for comparison to our data. To encourage such calculations and to facilitate comparisons with our experimental results, we include data tables as Supplemental Material [58].

\section{ACKNOWLEDGMENTS}

We thank the staff of the Collider-Accelerator and Physics Departments at Brookhaven National Laboratory and the staff of the other PHENIX participating institutions for their vital contributions. We also thank J. C. Collins for valuable discussions regarding the interpretation of these results. We acknowledge support from the Office of Nuclear Physics in the Office of Science of the Department of Energy, the National Science Foundation, Abilene Christian University Research Council, Research Foundation of SUNY, and Dean of the College of Arts and Sciences, Vanderbilt University (United States); Ministry of Education, Culture, Sports, Science, and Technology and the Japan Society for the Promotion of Science (Japan); Conselho Nacional de Desenvolvimento Científico e Tecnológico and Fundação de Amparo à Pesquisa do Estado de São Paulo (Brazil); Natural Science Foundation of China (People's Republic of China); Croatian Science Foundation and Ministry of Science and Education (Croatia); Ministry of Education, Youth and Sports (Czech Republic); Centre National de la Recherche Scientifique, Commissariat à l'Énergie Atomique, and Institut National de Physique Nucléaire et de Physique des Particules (France); Bundesministerium für Bildung und Forschung, Deutscher Akademischer Austausch Dienst, and Alexander von Humboldt Stiftung (Germany); J. Bolyai Research Scholarship, EFOP, the New National Excellence Program (ÚNKP), NKFIH, and OTKA (Hungary); Department of Atomic Energy and Department of Science and Technology (India); Israel Science Foundation (Israel); Basic Science Research Program through NRF of the Ministry of Education (Korea); Physics Department, Lahore University of Management Sciences (Pakistan); Ministry of Education and Science, Russian Academy of Sciences, Federal Agency of Atomic Energy (Russia); VR and Wallenberg Foundation (Sweden); the U.S. Civilian Research and Development Foundation for the Independent States of the Former Soviet Union; the Hungarian American Enterprise Scholarship Fund; the US-Hungarian Fulbright Foundation; and the US-Israel Binational Science Foundation. 
[1] A. Airapetian et al. (HERMES Collaboration), Single-Spin Asymmetries in Semi-Inclusive Deep-Inelastic Scattering on a Transversely Polarized Hydrogen Target, Phys. Rev. Lett. 94, 012002 (2005).

[2] C. Adolph et al. (COMPASS Collaboration), Sivers asymmetry extracted in SIDIS at the hard scales of the Drell-Yan process at COMPASS, Phys. Lett. B 770, 138 (2017).

[3] J. Huang et al. (Jefferson Lab Hall A Collaboration), BeamTarget Double Spin Asymmetry $A_{L T}$ in Charged Pion Production from Deep Inelastic Scattering on a Transversely Polarized He-3 Target at $1.4<Q^{2}<2.7 \mathrm{GeV}^{2}$, Phys. Rev. Lett. 108, 052001 (2012).

[4] L. Y. Zhu et al. (NuSea Collaboration), Measurement of Angular Distributions of Drell-Yan Dimuons in $p+p$ Interactions at 800-GeV/c, Phys. Rev. Lett. 102, 182001 (2009).

[5] M. Guanziroli et al. (NA10 Collaboration), Angular distributions of muon pairs produced by negative pions on deuterium and tungsten, Z. Phys. C 37, 545 (1988).

[6] R. Seidl et al. (Belle Collaboration), Measurement of Azimuthal Asymmetries in Inclusive Production of Hadron Pairs in $e^{+} e^{-}$Annihilation at Belle, Phys. Rev. Lett. 96, 232002 (2006).

[7] J. P. Lees et al. (BABAR Collaboration), Measurement of Collins asymmetries in inclusive production of charged pion pairs in $e^{+} e^{-}$annihilation at BABAR, Phys. Rev. D 90, 052003 (2014).

[8] C. Adolph et al. (COMPASS Collaboration), Collins and Sivers asymmetries in muonproduction of pions and kaons off transversely polarised protons, Phys. Lett. B 744, 250 (2015).

[9] G. Aad et al. (ATLAS Collaboration), Measurement of the jet fragmentation function and transverse profile in protonproton collisions at a center-of-mass energy of $7 \mathrm{TeV}$ with the ATLAS detector, Eur. Phys. J. C 71, 1795 (2011).

[10] B. B. Abelev et al. (ALICE Collaboration), Charged jet cross sections and properties in proton-proton collisions at $\sqrt{s}=7 \mathrm{TeV}$, Phys. Rev. D 91, 112012 (2015).

[11] L. Adamczyk et al. (STAR Collaboration), Azimuthal transverse single-spin asymmetries of inclusive jets and charged pions within jets from polarized-proton collisions at $\sqrt{s}=500 \mathrm{GeV}$, Phys. Rev. D 97, 032004 (2018).

[12] L. Adamczyk et al. (STAR Collaboration), Transverse spindependent azimuthal correlations of charged pion pairs measured in $p^{\uparrow}+p$ collisions at $\sqrt{s}=500 \mathrm{GeV}$, Phys. Lett. B 780, 332 (2018).

[13] C. A. Aidala, S. D. Bass, D. Hasch, and G. K. Mallot, The spin structure of the nucleon, Rev. Mod. Phys. 85, 655 (2013).

[14] D. W. Sivers, Single spin production asymmetries from the hard scattering of point-like constituents, Phys. Rev. D 41, 83 (1990).

[15] D. W. Sivers, Hard scattering scaling laws for single spin production asymmetries, Phys. Rev. D 43, 261 (1991).

[16] J. C. Collins, Leading twist single transverse-spin asymmetries: Drell-Yan and deep inelastic scattering, Phys. Lett. B 536, 43 (2002).

[17] L. Adamczyk et al. (STAR Collaboration), Measurement of the Transverse Single-Spin Asymmetry in $p^{\uparrow}+p \rightarrow W^{ \pm} / Z^{0}$ at RHIC, Phys. Rev. Lett. 116, 132301 (2016).
[18] M. Aghasyan et al. (COMPASS Collaboration), First Measurement of Transverse-Spin-Dependent Azimuthal Asymmetries in the Drell-Yan Process, Phys. Rev. Lett. 119, 112002 (2017).

[19] C. J. Bomhof, P. J. Mulders, and F. Pijlman, The construction of gauge-links in arbitrary hard processes, Eur. Phys. J. C 47, 147 (2006).

[20] J. C. Collins, 2-soft-gluon exchange and factorization breaking, arXiv:0708.4410.

[21] J. C. Collins and J. W. Qiu, $k_{T}$ factorization is violated in production of high-transverse-momentum particles in hadron-hadron collisions, Phys. Rev. D 75, 114014 (2007).

[22] T. C. Rogers and P. J. Mulders, No generalized TMDfactorization in hadro-production of high transverse momentum hadrons, Phys. Rev. D 81, 094006 (2010).

[23] S. Catani, D. de Florian, and G. Rodrigo, Space-like (versus time-like) collinear limits in QCD: Is factorization violated?, J. High Energy Phys. 07 (2012) 026.

[24] J. R. Forshaw, M. H. Seymour, and A. Siodmok, On the breaking of collinear factorization in QCD, J. High Energy Phys. 11 (2012) 066.

[25] B. B. Abelev et al. (ALICE Collaboration), Multiplicity dependence of the average transverse momentum in $\mathrm{pp}$, $\mathrm{p}-\mathrm{Pb}$, and $\mathrm{Pb}-\mathrm{Pb}$ collisions at the LHC, Phys. Lett. B 727, 371 (2013).

[26] A. Ortiz Velasquez, P. Christiansen, E. Cuautle Flores, I. A. Maldonado Cervantes, and G. Paić, Color Reconnection and Flowlike Patterns in $p p$ Collisions, Phys. Rev. Lett. 111, 042001 (2013).

[27] B. Blok, C. D. Jkel, M. Strikman, and U. A. Wiedemann, Collectivity from interference, J. High Energy Phys. 12 (2017) 074.

[28] S. Chatrchyan et al. (CMS Collaboration), Probing color coherence effects in pp collisions at $\sqrt{s}=7 \mathrm{TeV}$, Eur. Phys. J. C 74, 2901 (2014).

[29] M. Aaboud et al. (ATLAS Collaboration), High- $E_{\mathrm{T}}$ isolated-photon plus jets production in $p p$ collisions at $\sqrt{s}=8 \mathrm{TeV}$ with the ATLAS detector, Nucl. Phys. B918, 257 (2017).

[30] G. Aad et al. (ATLAS Collaboration), Measurement of colour flow with the jet pull angle in $t \bar{t}$ events using the ATLAS detector at $\sqrt{s}=8 \mathrm{TeV}$, Phys. Lett. B 750, 475 (2015).

[31] M. D. Schwartz, K. Yan, and H.X. Zhu, Factorization violation and scale invariance, Phys. Rev. D 97, 096017 (2018).

[32] A. Adare et al. (PHENIX Collaboration), Nonperturbativetransverse-momentum effects and evolution in dihadron and direct photon-hadron angular correlations in $p+p$ collisions at $\sqrt{s}=510 \mathrm{GeV}$, Phys. Rev. D 95, 072002 (2017).

[33] J. C. Collins, CSS equation, etc., follow from structure of TMD factorization, arXiv:1212.5974.

[34] A. Adare et al. (PHENIX Collaboration), High $p_{T}$ direct photon and $\pi^{0}$ triggered azimuthal jet correlations and measurement of $k_{T}$ for isolated direct photons in $p+p$ collisions at $\sqrt{s}=200 \mathrm{GeV}$, Phys. Rev. D 82, 072001 (2010).

[35] K. Adcox et al. (PHENIX Collaboration), PHENIX central arm tracking detectors, Nucl. Instrum. Methods Phys. Res., Sect. A 499, 489 (2003). 
[36] L. Aphecetche et al. (PHENIX Collaboration), PHENIX calorimeter, Nucl. Instrum. Methods Phys. Res., Sect. A 499, 521 (2003).

[37] A. Adare et al. (PHENIX Collaboration), Direct-photon production in $p+p$ collisions at $\sqrt{s}=200 \mathrm{GeV}$ at midrapidity, Phys. Rev. D 86, 072008 (2012).

[38] A. Adare et al. (PHENIX Collaboration), Inclusive cross section and double helicity asymmetry for $\pi^{0}$ production in $p+p$ collisions at $\sqrt{s}=62.4 \mathrm{GeV}$, Phys. Rev. D 79, 012003 (2009).

[39] A. Adare et al. (PHENIX Collaboration), Cross section and double helicity asymmetry for $\eta$ mesons and their comparison to neutral pion production in $p+p$ collisions at $\sqrt{s}=200 \mathrm{GeV}$, Phys. Rev. D 83, 032001 (2011).

[40] S. S. Adler et al. (PHENIX Collaboration), Measurement of Transverse Single-Spin Asymmetries for Mid-Rapidity Production of Neutral Pions and Charged Hadrons in Polarized $p+p$ Collisions at $\sqrt{s}=200 \mathrm{GeV}$, Phys. Rev. Lett. 95, 202001 (2005).

[41] S. S. Adler et al. (PHENIX Collaboration), PHENIX on-line and off-line computing, Nucl. Instrum. Methods Phys. Res., Sect. A 499, 593 (2003).

[42] A. Adare et al. (PHENIX Collaboration), Double-helicity dependence of jet properties from dihadrons in longitudinally polarized $p+p$ collisions at $\sqrt{s}=200 \mathrm{GeV}$, Phys. Rev. D 81, 012002 (2010).

[43] S. S. Adler et al. (PHENIX Collaboration), Jet properties from dihadron correlations in $p+p$ collisions at $\sqrt{s}=$ 200 GeV, Phys. Rev. D 74, 072002 (2006).

[44] A. Adare et al. (PHENIX Collaboration), Photon-hadron jet correlations in $p+p$ and $\mathrm{Au}+\mathrm{Au}$ collisions at $\sqrt{s}=200 \mathrm{GeV}$, Phys. Rev. C 80, 024908 (2009).

[45] A. M. Sirunyan et al. (CMS Collaboration), Study of jet quenching with isolated-photon + jet correlations in $\mathrm{PbPb}$ and pp collisions at $\sqrt{s_{\mathrm{NN}}}=5.02 \mathrm{TeV}$, Phys. Lett. B 785, 14 (2018).

[46] A. S. Ito et al., Measurement of the continuum of dimuons produced in high-energy proton- nucleus collisions, Phys. Rev. D 23, 604 (1981).

[47] F. Landry, R. Brock, P. M. Nadolsky, and C. P. Yuan, Tevatron Run-1 Z boson data and Collins-Soper-Sterman resummation formalism, Phys. Rev. D 67, 073016 (2003).
[48] A. V. Konychev and P. M. Nadolsky, Universality of the Collins-Soper-Sterman nonperturbative function in gauge boson production, Phys. Lett. B 633, 710 (2006).

[49] P. Schweitzer, T. Teckentrup, and A. Metz, Intrinsic transverse parton momenta in deeply inelastic reactions, Phys. Rev. D 81, 094019 (2010).

[50] C. A. Aidala, B. Field, L. P. Gamberg, and T. C. Rogers, Limits on transverse momentum dependent evolution from semi-inclusive deep inelastic scattering at moderate $Q$, Phys. Rev. D 89, 094002 (2014).

[51] P. Nadolsky, D. R. Stump, and C. P. Yuan, Semiinclusive hadron production at HERA: The effect of QCD gluon resummation, Phys. Rev. D 61, 014003 (1999); Erratum, Phys. Rev. D 64, 059903(E) (2001).

[52] L. Gamberg, A. Metz, D. Pitonyak, and A. Prokudin, Connections between collinear and transversemomentum-dependent polarized observables within the Collins-Soper-Sterman formalism, Phys. Lett. B 781, 443 (2018).

[53] J. Collins, L. Gamberg, A. Prokudin, T. C. Rogers, N. Sato, and B. Wang, Relating transverse momentum dependent and collinear factorization theorems in a generalized formalism, Phys. Rev. D 94, 034014 (2016).

[54] J. Collins and T. Rogers, Understanding the large-distance behavior of transverse-momentum-dependent parton densities and the Collins-Soper evolution kernel, Phys. Rev. D 91, 074020 (2015).

[55] A. Bacchetta, F. Delcarro, C. Pisano, M. Radici, and A. Signori, Extraction of partonic transverse momentum distributions from semi-inclusive deep-inelastic scattering, Drell-Yan and Z-boson production, J. High Energy Phys. 06 (2017) 081.

[56] T. C. Rogers, Extra spin asymmetries from the breakdown of transverse-momentum-dependent factorization in hadronhadron collisions, Phys. Rev. D 88, 014002 (2013).

[57] A. Schäfer and J. Zhou, Color entanglement for $\gamma$-jet production in polarized pA collisions, Phys. Rev. D 90, 094012 (2014).

[58] See Supplemental Material at http://link.aps.org/ supplemental/10.1103/PhysRevD.98.072004 for data tables showing numerical values for the experimental results. 